\title{
Effects of mulberry leaf extracts on activity and mRNA expression of five cytochrome P450 enzymes in rat
}

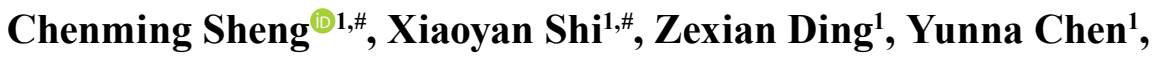 \\ Xiaoqian Shi', Yifan Wu', Wenjing Zhang', Weidong Chen ${ }^{1 *}$ \\ ${ }^{1}$ Pharmacokinetics Laboratory, School of Pharmacy, Anhui University of \\ Chinese Medicine, 001 Qianjiang Road, Hefei 230012, Anhui, China
}

\begin{abstract}
Adverse side effects of drug-drug interactions induced by human cytochrome P450 (CYP450) inhibition is an important consideration in drug discovery. Mulberry leaves are of broad popular use for food or remedy purposes, which is believed to contain substances that are beneficial for preventing and alleviating diabetes. However, there is a paucity of information about the effect of mulberry leaves on rat CYP450 enzymes activities and the mRNA expression levels in vivo. The present study aimed to investigate the effect of mulberry leaves on activities of rat CYP450 enzymes (CYP3A4, CYP2C8, CYP2C19, CYP2D6, and CYP1A2) through both probe-drug cocktail approach and real-time polymerase chain reaction (RT-qPCR). The pharmacokinetic results indicated that the aqueous extract of mulberry leaves (AML) exhibited induction effects on CYP3A4 activities, and AML exhibited inhibitory effects on CYP1A2, CYP2D6, and CYP2C8, while no obvious effect was observed on CYP2C19 activity. Additionally, the ethanol extract of mulberry leaves (EML) could induce the activities of CYP3A4. In addition, EML exhibited inhibitory effects on CYP1A2, CYP2D6, and CYP2C19, while no significant change in CYP2C8 activity was observed. Accordingly, the level of mRNA expression of five CYP enzymes were consistent with the result of pharmacokinetic. The results of our study may form a practical strategy for assessing CYP-mediated HDI.
\end{abstract}

Keywords: Mulberry leaves. Cytochrome P450. mRNA. Cocktail.

\section{INTRODUCTION}

Mulberry leaf (Folium mori) pertains to dried leaves of the Morus alba L. plant (family Moraceae) (Joh et al., 2015) known to be rich in flavonoids, alkaloids, polysaccharides, volatile oils, and other active components. The rapid increase in research interest on the potential therapeutic applications of mulberry leaves has led to successful characterization of the major pharmacological properties, including activities against dyslipidemia, diabetes, fatty liver disease and hypertension (Zhang et al., 2014; Gurukar

"Correspondence: Wei-Dong Chen. Pharmacokinetics Laboratory. School of Pharmacy. Anhui University of Chinese Medicine. 001 Qianjiang Road. Hefei 230012, Anhui, China. Tel: +86 15156091053. E-mail: wdchen@ ahtcm.edu.cn. ${ }^{\text {TThe }}$ authors contributed equally to this work
MSA, Chilkunda ND, 2018; Zheng et al., 2017; Wang et al., 2015). Owing to their multiple beneficial effects, mulberry leaves are commercially used in the formulation of nutraceuticals, tea supplements, cosmetics and functional foods in Asia.

The cytochrome P450 (CY P450) superfamily is responsible for metabolism of various endogenous compounds and xenobiotics (Zhou SF, Liu JP, Chowbay B, 2009; Guo YJ, Zheng SL, 2014). Among the several CYP450 isoforms identified to date, human CYP3A4, CYP1A2, CYP2D6, CYP2C19, and CYP2C8 metabolize over 90\% clinical drugs (Rendic S, Di Carlo FJ, 1997; Reginald EF, 2004). Clinically, inhibition or stimulation of CYP activity is a potential cause of the diverse pharmacokinetics of drugs, resulting in unexpected and even serious clinical herb-drug interactions (HDI). HDIs may lead to toxicity or poor efficacy of drugs 
and are a preventable cause of adverse drug events (ADE) (Liu et al., 2017; Cai et al., 2017). Clarification of the mechanisms by which mulberry leaves influence the activity of CYP isoenzymes may aid in reducing the potential risks of co-administration of herbal and conventional medicines, which are of significant concern.

Although several investigations have focused on mulberry leaves over the years, to our knowledge, none have determined their effects on CYP450 enzymes. For establishing safety of usage in clinical combinations, we evaluated the influence of mulberry leaf extracts on the activities of five CYP450 subtypes based on changes in pharmacokinetic parameters in an in vivo rat model using specific probe drugs (midazolam for Cyp3A4, phenacetin for Cyp1A2, dextromethorphan for Cyp2D6, omeprazole for Cyp2C19 and amodiaquine for Cyp2C8), compared to a control group. RT-qPCR analysis was employed to evaluate the effects of mulberry leaf on mRNA expression of the above CYP enzymes. A sensitive and specific ultraperformance liquid chromatography tandem mass spectrometry (UPLC-MS/MS) method was applied for simultaneous quantification of the concentrations of the five probe drugs via a single-run process. Our collective findings demonstrate that mulberry leaf extracts induce significant changes in liver CYP450 activity in vivo and should aid in determining and thus preventing the potential HDIs resulting from CYP450 enzyme induction or inhibition.

\section{MATERIAL AND METHODS}

\subsection{Chemicals and reagents}

Dried mulberry leaf was purchased from the Bozhou Chinese medicinal materials market and authenticated by Professor Nianjun Yu (Anhui University of Chinese Medicine). Midazolam, phenacetin, dextromethorphan, omeprazole, amodiaquine and the internal standard, glibenclamide with $>98.0 \%$ purity were supplied by the China National Institutes for Food and Drug Control. LC-MS grade acetonitrile, methanol and formic acid were obtained from Merck Company (Darmstadt, Germany). Ultrapure water (resistance $>18.2 \mathrm{~m} \Omega$ ) was prepared using a Millipore Milli-Q purification system (Bedford, USA). All other chemicals were of analytical grade and used without further purification.

\subsection{Apparatus}

All analyses was performed on a 1290 Series liquid chromatography column (Agilent Technologies, Germany) equipped with a quaternary pump, degasser, autosampler, thermostatted column compartment and an AB SCIEX Triple QuadTM 4500 mass spectrometer (Applied Biosystems) equipped with an atmospheric pressure chemical ionization source (APCI) controlled by Analyst software.

\subsection{Preparation of mulberry leaf extracts}

To prepare AML, dried leaf powder was extracted twice in water $(1: 25)$ at $70^{\circ} \mathrm{C}$. The supernatant fraction was concentrated in a rotary evaporator at $55^{\circ} \mathrm{C}$ under vacua and freeze-dried. The dry powder obtained was stored at $-20{ }^{\circ} \mathrm{C}$. To obtain EML, dried leaf powder samples were extracted twice with $95 \%$ ethanol $(1: 15)$ at $85^{\circ} \mathrm{C}$. The supernatant was concentrated in a rotary evaporator at $55^{\circ} \mathrm{C}$ under vacuum and freeze-dried, followed by storage at $-20^{\circ} \mathrm{C}$.

\subsection{Experimental animals}

Male Sprague-Dawley rats $(200 \pm 20 \mathrm{~g})$ were obtained from the Laboratory Animal Center of Anhui Medical University (Hefei, Anhui; China Permit Number SCXK 2011-0002). Animal studies were strictly performed according to the guidelines provided by the Animal Ethics Committee (Anhui University of Chinese Medicine, China). Animals were housed in cages at $23-25^{\circ} \mathrm{C}$ with free access to a regular rodent diet and water.

\subsection{Drug administration and sampling}

Forty-eight male rats were randomly divided into eight groups ( $\mathrm{n}=6$ ): control (saline, NS), low-dosage AML (AML-L, $1 \mathrm{~g} / \mathrm{kg}$ ), medium-dosage AML (AML-M, 2 $\mathrm{g} / \mathrm{kg}$ ) and high-dosage AML (AML-H, $4 \mathrm{~g} / \mathrm{kg}$ ), control (0.5\% CMC-Na, CMC), low-dosage EML (EML-L, $1 \mathrm{~g} /$ $\mathrm{kg}$ ), medium-dosage EML (EML-M, $2 \mathrm{~g} / \mathrm{kg}$ ) and highdosage EML (EML-H, $4 \mathrm{~g} / \mathrm{kg}$ ). EML (dissolved in $0.5 \%$ CMC-Na solution) and AML (dissolved in saline) were orally administered for 14 consecutive days while the control groups received CMC-Na/saline for 14 days. After $30 \mathrm{~min}$ of the final treatment, $1 \mathrm{mg} / \mathrm{kg}(2.5 \mathrm{~mL} / \mathrm{kg})$ midazolam, phenacetin, dextromethorphan, omeprazole or amodiaquine was administered intravenously via the 
tail vein. Blood samples $(0.25 \mathrm{~mL})$ were collected in heparinized tubes from the fundus venous plexus predose $(0 \mathrm{~h})$ and at $0.05,0.08,0.17,0.25,0.5,1,2,4,6,8$ and $12 \mathrm{~h}$. Plasma samples were immediately separated via centrifugation at $3500 \mathrm{rpm}$ for $10 \mathrm{~min}$ and stored at $-80{ }^{\circ} \mathrm{C}$ for subsequent analyses.

\subsection{Sample preparation}

All samples $(100 \mu \mathrm{L})$ were spiked with the internal standard glibenclamide (final concentration: $100 \mathrm{ng} / \mathrm{mL}$ ) and mixed with $300 \mu \mathrm{L}$ methanol. The resulting mixture was vortexed for $5 \mathrm{~min}$ and centrifuged at 12,000 rpm for $10 \mathrm{~min}$. The supernatant injected into the UPLC-MS/MS system for analysis.

\subsection{Chromatographic conditions}

Chromatographic separation was conducted on a $2.1 \mathrm{~mm} \times 50 \mathrm{~mm}, 1.7 \mu \mathrm{m}$ particle, ACQUITY UPLC BEH $\mathrm{C} 18$ column at $30^{\circ} \mathrm{C}$. The mobile phase consisted of (A) $0.1 \%$ formic acid in water and (B) acetonitrile with a total analysis time of $4.5 \mathrm{~min}$ and gradient elution of
$0.01-1 \min (10-80 \% \mathrm{~B}), 1-1.3 \min (80-80 \% \mathrm{~B}), 1.3-2$ $\min (80-95 \%$ B), 2-3 $\min (95-90 \%$ B), 3-3.5 min (90$90 \%$ B), 3.5-4 min (90-10\% B), 4-4.5 min (10-10\% B) employed. The flow rate was $0.2 \mathrm{~mL} / \mathrm{min}$ and injection volume was $2 \mu \mathrm{L}$.

Mass spectrometric detection was conducted in the positive mode. Multiple reaction monitoring (MRM) conditions were defined as follows: capillary voltage, $2 \mathrm{kV}$; nebulizer gas (N2), $650 \mathrm{~L} / \mathrm{h}$; source temperature, $450{ }^{\circ} \mathrm{C}$; desolvation temperature, $500{ }^{\circ} \mathrm{C}$. Fragmentation transition and MS parameters are listed in Table I.

$R N A$ extraction and RT-qPCR determination of gene expression in liver Rat livers were removed after the final treatment, washed in ice-cold saline solution, blotted dry and stored at $-80{ }^{\circ} \mathrm{C}$.

Gene expression in liver was examined as described previously (Lu et al., 2018). The quality and quantity of extracted mRNA was determined by measurement with a microplate reader (Thermo Fisher Scientific, USA). Expression of the target genes Cyp3a1, Cypla2, Cyp2d2, Cyp2c11, and Cyp2c7 was analyzed using the housekeeping gene, GAPDH, for data normalization (Table II).

TABLE I - MRM transitions and collision energy and for the detection of the CYP probe drugs and internal standard

\begin{tabular}{|c|c|c|c|c|c|c|}
\hline (Compound) & $\begin{array}{l}\text { Q1(mass) } \\
(\mathbf{m} / \mathbf{z})\end{array}$ & $\begin{array}{c}\text { Q3 (mass) } \\
(\mathrm{m} / \mathbf{z})\end{array}$ & $\mathbf{D P} / \mathbf{V}$ & $\mathbf{E P} / \mathbf{V}$ & $\mathrm{CE} / \mathrm{eV}$ & $\mathrm{CXP} / \mathrm{V}$ \\
\hline Midazolam & 326.1 & 290.6 & 102.6 & 10 & 35.8 & 11 \\
\hline Phenacetin & 180.1 & 109.9 & 81.0 & 10 & 29.0 & 11 \\
\hline Dextromethorphan & 272.1 & 171.0 & 111.0 & 10 & 53.6 & 11 \\
\hline Omeprazole & 346.1 & 197.9 & 58.3 & 10 & 14.5 & 11 \\
\hline Amodiaquine & 356.3 & 283.0 & 80.0 & 10 & 25.0 & 11 \\
\hline Glibenclamide & 493.9 & 169.1 & 115.0 & 10 & 55.0 & 11 \\
\hline
\end{tabular}


TABLE II - Oligonucleotide primers used for real-time RT-qPCR

\begin{tabular}{lcc} 
Target & Forward primer sequence & Reverse primer sequence \\
\hline Cyp3a1 & TGCCATCACGGACACAGA & ATCTCTTCCACTCCTCATCCTTAG \\
\hline Cyp1a2 & CATCTTTGGAGCTGGATTTG & CCATTCAGGAGGTGTCC \\
Cyp2d2 & GAAGGAGAGCTTTGGAGAGGA & AGAATTGGGATTGCGTTCAG \\
\hline Cyp2c11 & GGAGGAACTGAGGAAGAGCA & AATGGAGCATATCACATTGCAG \\
\hline Cyp2c7 & TGTGAAGAACATCAGCCAATCCT & CACGGTCCTCAATGTTCCTTTT \\
\hline GAPDH & TGCTGAGTATGTCGTGGAG & GTCTTCTGAGTGGCAGTGAT \\
\hline
\end{tabular}

\subsection{Statistical analysis}

Data were analyzed with GraphPad Prism 5.0 software (GraphPad Inc., San Diego, CA, USA) and presented as means $\pm \mathrm{SD}$, with the level of significance set at $\mathrm{P}<0.05$. One-way ANOVA followed by Bonferroni test was applied to compare between-group differences.

\section{RESULTS}

\subsection{UPLC-MS/MS method validation}

The UPLC-MS/MS method was validated by assessment of specificity, calibration curve, precision, accuracy, matrix effect and stability profiles of the five probe substrates. Through comparing the retention times from chromatograms of blank plasma, blank plasma spiked with the five probe drugs and IS and a plasma sample collected after tail vein injection of probe drugs in rats, specificity was evaluated. As depicted in Figure 1, they had a better separation effect.

The standard curves and concentration ranges of each probe drug were $\mathrm{y}=0.061 \mathrm{x}+0.0689$ for midazolam $(5-3000$ $\mathrm{ng} / \mathrm{mL}), \mathrm{y}=0.1286 \mathrm{x}+0.0327$ for phenacetin $(2-4000 \mathrm{ng} /$ $\mathrm{mL}), \mathrm{y}=0.1063 \mathrm{x}+0.137$ for dextromethorphan $(5-1500$ $\mathrm{ng} / \mathrm{mL}) ; \mathrm{y}=0.1092 \mathrm{x}-0.0816$ for omeprazole $(5-6000 \mathrm{ng} /$ $\mathrm{mL}), \mathrm{y}=0.2193 \mathrm{x}+0.1454$ for amodiaquine $(1-500 \mathrm{ng} / \mathrm{mL})$ $(\mathrm{x}=$ concentration of each probe drug; $\mathrm{y}=$ peak area ratio of each probe drug vs. internal standard), with the correlation coefficients of calibration curves determined as $>0.99$.

Using quality control (QC) samples at low (LQC), medium (MQC), and high (HQC) concentrations, intraday and inter-day precision values of all the probe drugs were evaluated as $<10.20 \%$ and $14.7 \%$, respectively. Accuracy expressed for all QC levels was within $10.12 \%$.

The matrix effects in the LQC, MQC and HQC groups (mean $\pm \mathrm{RSD}$ ) were 102.53, 98.71 and $93.23 \%$ with RSD values of 7.47, 7.30 and $2.70 \%(n=6)$ for midazolam, 99.63, 92.22 and $102.11 \%$ with RSD values of 5.47, 5.30 and $6.76 \%$ for phenacetin, $94.13,93.64$ and $92.67 \%$ with RSD values of $2.38,6.15$ and $3.72 \%$ for dextromethorphan, $93.41,96.67$ and $94.15 \%$ with RSD values of $3.69,4.12$ and $5.03 \%$ for omeprazole, and 90.17 , 93.16 and $95.60 \%$ with RSD values of 5.18, 6.18 and $5.47 \%$ for amodiaquine.

The stability of each probe drug in plasma was investigated through analyzing three replicates for all QC levels under processing conditions and manifold storage. The results (range, $91.10-104.53 \%$; precision $<15.00 \%$ ) for short-term stability ( $2 \mathrm{~h}$ at room temperature), long-term stability $\left(7\right.$ days at $\left.-80^{\circ} \mathrm{C}\right)$, stability in the autosampler for $12 \mathrm{~h}$ and freeze-thaw stability ( 3 cycles) met the requirements of the test. 
A


B
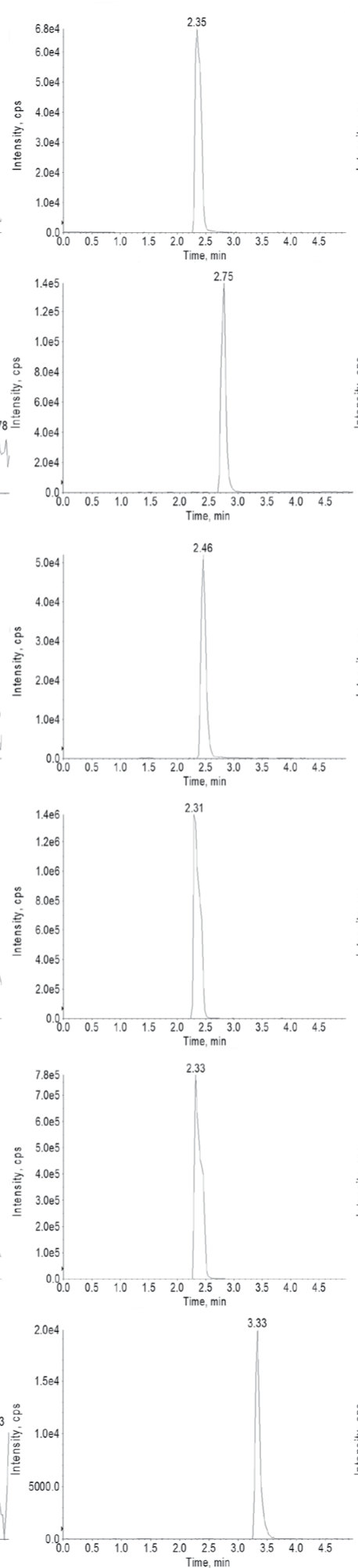

C
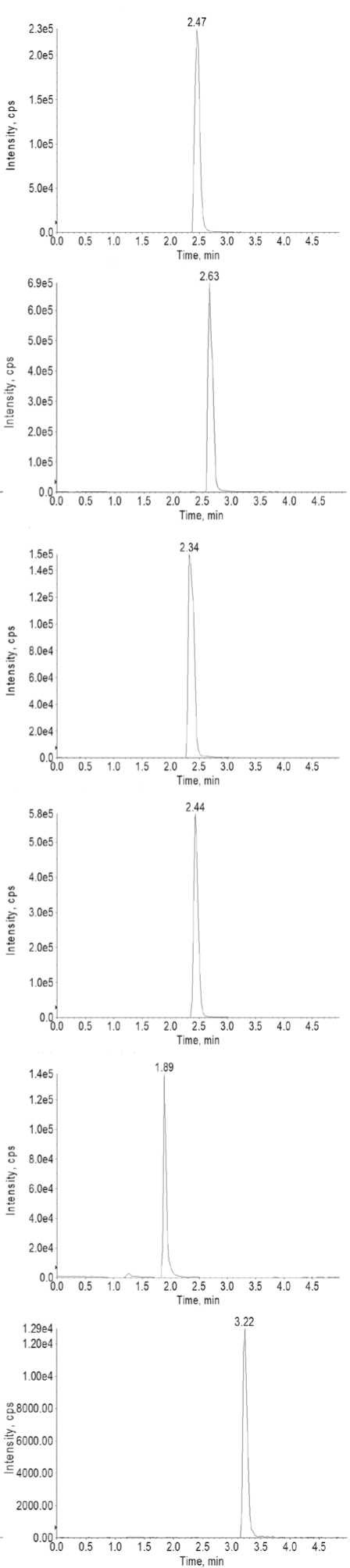

FIGURE 1 - LC-MS/MS chromatograms of probes and the internal standard in SD rats plasma. A. Blank plasma; B. Blank plasma + Probe substrate +Internal standard; C. Probe substrate after tail vein injection of probe substrate in rat +Internal standard; (I. midazolam; II. phenacetin; III. dextromethorphan; IV. omeprazole; V. amodiaquine; VI. glibenclamide.). 


\subsection{Effects of mulberry leaf extracts on CYP activities in rats}

\subsubsection{Effects of mulberry leaf extracts on CYP3A4}

The pharmacokinetic profiles of midazolam after mulberry leaf extract treatment were used to assess CYP3A4 activity (Table III). The mean plasma concentration-time profiles of midazolam in control and mulberry leaf extract groups are shown in
Figure 2. After pretreatment with all doses of AML, the main pharmacokinetic parameters $\left(\mathrm{AUC}_{(0-\mathrm{t})}\right.$ and $\left.\mathrm{AUC}_{(0-\infty)}\right)$ of midazolam in rats were significantly decreased, compared to those of the NS group, while CL was significantly increased. A similar trend of pharmacokinetic parameters was observed in the EML treatment groups, indicating that the metabolism of midazolam is highly accelerated. Thus, both AML and EML appear to have the potential to stimulate rat hepatic CYP3A4 activity in vivo.

TABLE III - Main pharmacokinetic parameters of midazolam in rats $(n=6$, mean \pm SD)

\begin{tabular}{|c|c|c|c|c|}
\hline NS & $246.862 \pm 20.804$ & $262.309 \pm 25.037$ & $122.661 \pm 11.591$ & $0.004 \pm 0.001$ \\
\hline AML-M & $78.327 \pm 3.069 * *$ & $85.567 \pm 4.179^{* *}$ & $194.052 \pm 24.701$ & $0.012 \pm 0.001^{* *}$ \\
\hline AML-H & $70.678 \pm 6.948^{* *}$ & $85.639 \pm 7.859^{* *}$ & $169.161 \pm 29.361$ & $0.012 \pm 0.001 * *$ \\
\hline EML-M & $97.598 \pm 6.746^{* *}$ & $105.950 \pm 6.392 * *$ & $159.491 \pm 12.941$ & $0.009 \pm 0.001^{* *}$ \\
\hline EML-H & $98.306 \pm 10.255^{* *}$ & $108.325 \pm 10.905^{* *}$ & $168.936 \pm 12.391$ & $0.009 \pm 0.001 * *$ \\
\hline
\end{tabular}

* Significantly different with $P<0.05$ versus control group.

** Significantly different with $P<0.01$ versus control group. 

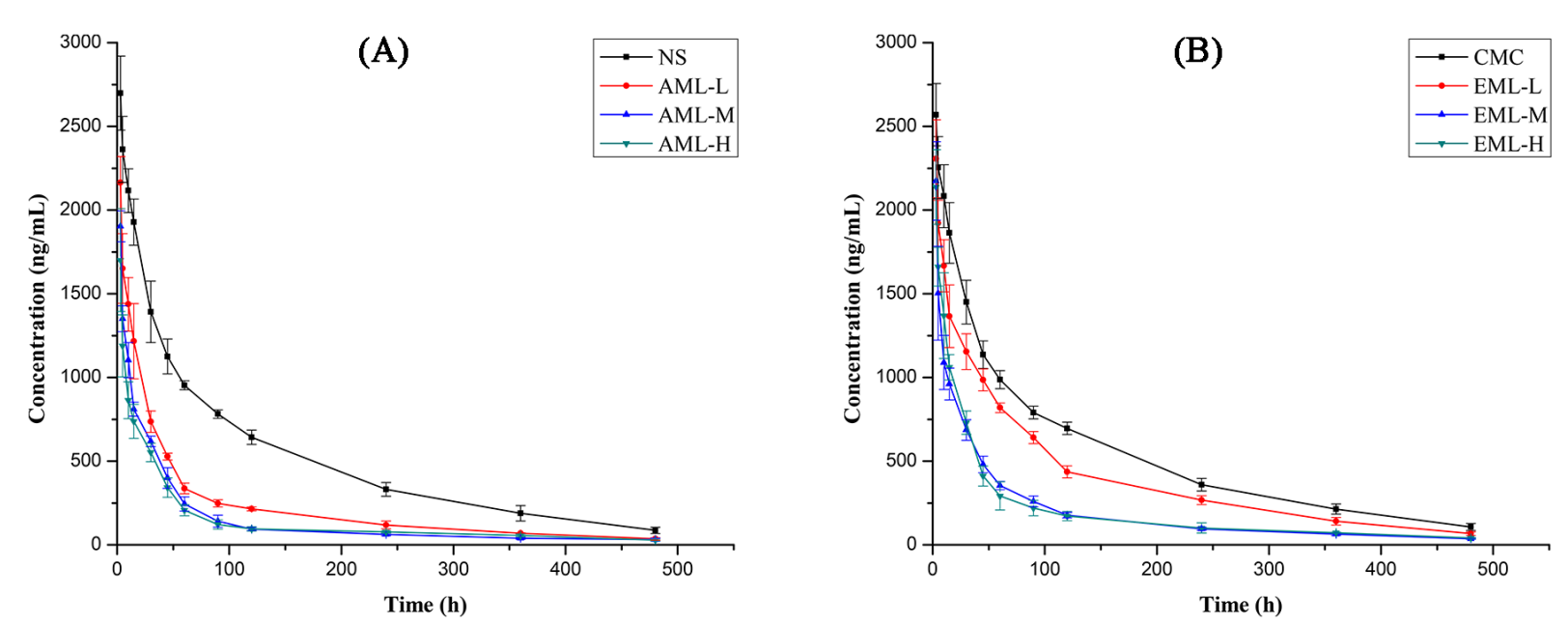

FIGURE 2 - Mean plasma concentration-time profiles of midazolam in rats pre-treated with AML (A) and EML (B).

\subsubsection{Effects of mulberry leaf extracts on CYP1A2}

The effects of three doses of mulberry leaf extracts on the pharmacokinetic profiles of phenacetin in rats are described in Table IV. The mean plasma concentrationtime curves of phenacetin in control and mulberry leaf extract groups are presented in Figure 3. After intragastric administration of AML for 14 days, $\mathrm{AUC}_{(0-t)}$ and $\mathrm{AUC}_{(0-}$ ${ }_{\infty}$ values of phenacetin in AML-L were significantly increased (by $43.64 \%$ and $44.94 \%$, respectively) and CL was significantly decreased (by $30.30 \%$ ), compared with the corresponding values in NS. In the AML-M group, $\operatorname{AUC}(0-\mathrm{t})$ and $\mathrm{AUC}_{(0-\infty)}$ of phenacetin were increased to a higher extent (by $99.57 \%$ and $101.27 \%$, respectively), and CL was more significantly decreased (by $45.45 \%$ ), compared with the corresponding values in $\mathrm{NS}$. $\mathrm{AUC}_{(0-\mathrm{t})}$ and $\mathrm{AUC}_{(0-\infty)}$ of phenacetin in AML-H were significantly increased (by $60.34 \%$ and $60.62 \%$, respectively) and
CL was significantly decreased (by $36.36 \%$ ), compared with the corresponding values in NS. Our results clearly demonstrate that the metabolism of phenacetin in all groups is decreased, indicating significant inhibition of CYP2C9 activity by AML in rat. Interestingly, based on the main pharmacokinetic parameters, the inhibitory effect of AML on CYP1A2 was higher in the AML-M than AML-H group.

On the other hand, in the EML treatment groups, $\mathrm{AUC}_{(0-\mathrm{t})}$ of phenacetin in EML-M and EML-H groups was significantly increased (by $53.17 \%$ and $52.74 \%$, respectively) along with $\mathrm{AUC}_{(0-\infty)}$ (by $53.44 \%$ and $52.45 \%$, respectively) and CL was significantly decreased (by $35.29 \%$ and $32.35 \%$, respectively), compared with the corresponding values in $\mathrm{CMC}$. However, the pharmacokinetic profiles $\left(\mathrm{AUC}_{(0-\mathrm{t})}, \mathrm{AUC}_{(0-\infty)}\right.$ and $\left.\mathrm{CL}\right)$ of phenacetin in EML-L and CMC groups were not significantly different.

TABLE IV - Main pharmacokinetic parameters of phenacetin in rats $(n=6$, mean \pm SD)

\begin{tabular}{|c|c|c|c|c|}
\hline Group & $\begin{array}{c}\text { AUC(0-t) } \\
(\mathrm{mg} / \mathrm{L} \cdot \mathbf{m i n})\end{array}$ & $\begin{array}{c}\operatorname{AUC}(0-\infty) \\
(\mathrm{mg} / \mathbf{L} \cdot \mathbf{m i n})\end{array}$ & $\begin{array}{c}t_{1 / 2} \\
(\mathrm{~min})\end{array}$ & $\begin{array}{c}\mathrm{CL} \\
(\mathrm{L} / \mathrm{min} / \mathrm{kg})\end{array}$ \\
\hline NS & $29.707 \pm 2.197$ & $29.886 \pm 2.250$ & $17.170 \pm 1.616$ & $0.033 \pm 0.003$ \\
\hline AML-L & $42.670 \pm 4.464^{* *}$ & $43.316 \pm 5.149^{* *}$ & $22.187 \pm 4.189$ & $0.023 \pm 0.003 * *$ \\
\hline
\end{tabular}


TABLE IV - Main pharmacokinetic parameters of phenacetin in rats $(n=6$, mean $\pm \mathrm{SD})$

\begin{tabular}{|c|c|c|c|c|}
\hline Group & $\begin{array}{c}\operatorname{AUC}(0-t) \\
(\mathbf{m g} / \mathbf{L} \cdot \min )\end{array}$ & $\begin{array}{c}\operatorname{AUC}(0-\infty) \\
(\mathrm{mg} / \mathbf{L} \cdot \min )\end{array}$ & $\begin{array}{c}t_{1 / 2} \\
(\mathrm{~min})\end{array}$ & $\begin{array}{c}C L \\
(\mathrm{~L} / \mathrm{min} / \mathrm{kg})\end{array}$ \\
\hline AML-M & $59.285 \pm 9.332^{* *}$ & $60.153 \pm 9.450^{* *}$ & $29.413 \pm 4.849$ & $0.018 \pm 0.005^{* *}$ \\
\hline AML-H & $47.632 \pm 3.849^{* *}$ & $48.003 \pm 4.043^{* *}$ & $20.538 \pm 2.166$ & $0.021 \pm 0.003^{* *}$ \\
\hline $\mathrm{CMC}$ & $29.329 \pm 1.737$ & $29.509 \pm 1.616$ & $17.540 \pm 4.312$ & $0.034 \pm 0.002$ \\
\hline EML-L & $29.051 \pm 3.199$ & $29.358 \pm 3.415$ & $20.267 \pm 2.088$ & $0.034 \pm 0.004$ \\
\hline EML-M & $44.924 \pm 4.047^{* *}$ & $45.278 \pm 4.084^{* *}$ & $19.916 \pm 3.144$ & $0.022 \pm 0.002^{* *}$ \\
\hline EML-H & $44.796 \pm 6.497^{* *}$ & $44.986 \pm 6.659^{* *}$ & $17.152 \pm 4.270$ & $0.023 \pm 0.003^{* *}$ \\
\hline
\end{tabular}

* Significantly different with $P<0.05$ versus control group.

** Significantly different with $P<0.01$ versus control group.
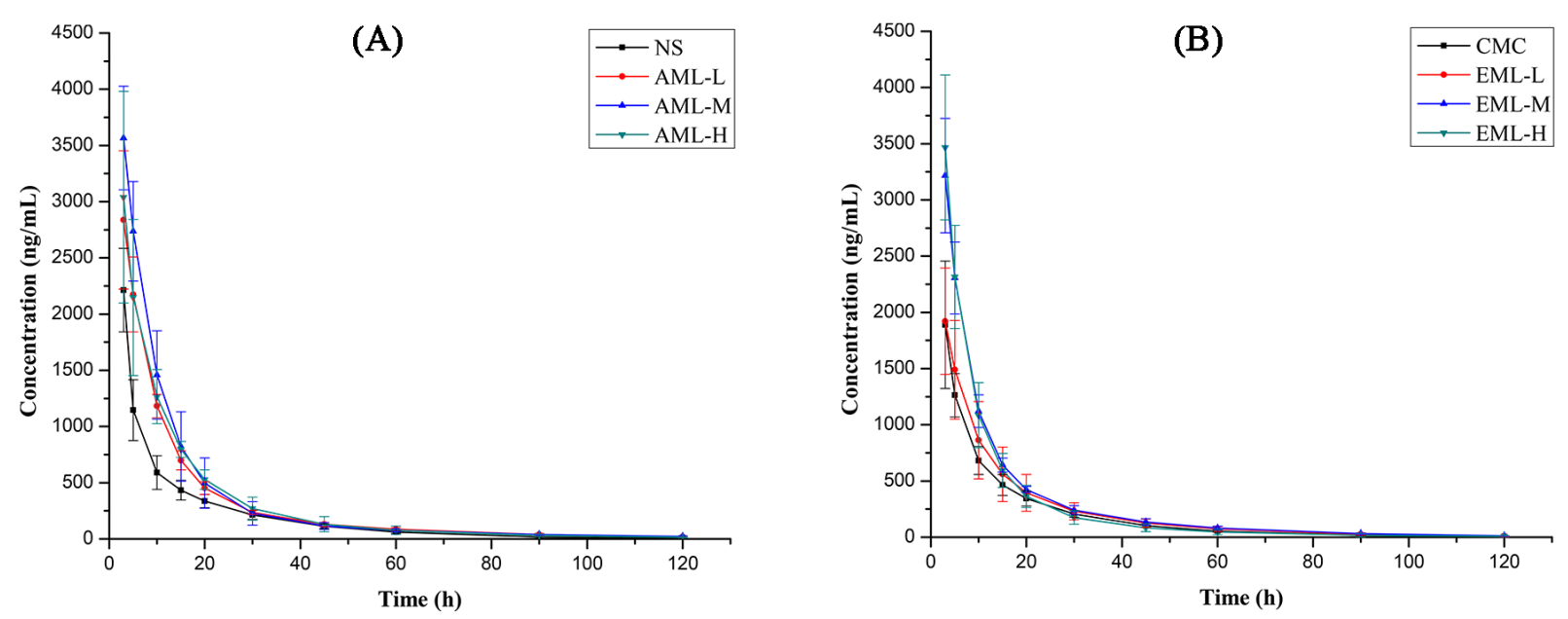

FIGURE 3 - Mean plasma concentration-time profiles of phenacetin in rats pre-treated with AML (A) and EML (B).

\subsubsection{Effects of mulberry leaf extracts on CYP2D6}

The effects of various doses of mulberry leaf extract on the pharmacokinetics of dextromethorphan in rats are presented in Table V. The mean plasma concentration-time profiles of dextromethorphan in all three groups are shown in Figure 4. Our results disclosed a significant increase in $\mathrm{AUC}_{(0-\mathrm{t})}$ of dextromethorphan after pretreatment with EML-L, EML-M, and EML-H doses (by 5.72\%, 69.74\% and 93.69\%, respectively), compared with CMC. Additionally,
$\mathrm{AUC}_{(0-\infty)}$ of dextromethorphan was significantly increased after pretreatment with all three doses (by $8.10 \%, 72.79 \%$, and $94.81 \%$, respectively) and CL significantly decreased (by $4.76 \%, 42.86 \%$, and $47.62 \%$, respectively) relative to $\mathrm{CMC}$, supporting an inhibitory effect of EML on rat hepatic CYP2D6. On the other hand, after pretreatment with AML-M and AML-H, the main parameters of dextromethorphan $\left(\mathrm{AUC}_{(0-\mathrm{t})}\right.$ and $\left.\mathrm{AUC}_{(0-\infty)}\right)$ were markedly increased while $\mathrm{CL}$ was decreased, compared with $\mathrm{CMC}$. For the AML-L group, no apparent effects were evident. 
TABLE V - Main pharmacokinetic parameters of dextromethorphan in rats $(\mathrm{n}=6$, mean $\pm \mathrm{SD})$

\begin{tabular}{|c|c|c|c|c|}
\hline Group & $\begin{array}{c}\mathrm{AUC}(0-\mathrm{t}) \\
(\mathrm{mg} / \mathrm{L} \cdot \mathrm{min})\end{array}$ & $\begin{array}{c}\operatorname{AUC}(0-\infty) \\
(\mathrm{mg} / \mathrm{L} \cdot \min )\end{array}$ & $\begin{array}{c}\mathbf{t}_{1 / 2} \\
(\mathbf{m i n})\end{array}$ & $\begin{array}{c}\mathrm{CL} \\
(\mathrm{L} / \mathrm{min} / \mathrm{kg})\end{array}$ \\
\hline NS & $42.495 \pm 1.820$ & $44.595 \pm 2.400$ & $117.489 \pm 16.520$ & $0.022 \pm 0.001$ \\
\hline AML-L & $43.245 \pm 1.737$ & $45.692 \pm 1.865$ & $119.841 \pm 11.085$ & $0.022 \pm 0.001$ \\
\hline AML-M & $58.792 \pm 3.360^{* *}$ & $62.954 \pm 3.897^{* *}$ & $169.067 \pm 21.404$ & $0.016 \pm 0.001^{* *}$ \\
\hline AML-H & $66.820 \pm 5.556^{* *}$ & $72.160 \pm 4.703^{* *}$ & $170.883 \pm 12.441$ & $0.013 \pm 0.001^{* *}$ \\
\hline $\mathrm{CMC}$ & $44.474 \pm 1.910$ & $46.743 \pm 2.711$ & $126.355 \pm 19.338$ & $0.021 \pm 0.001$ \\
\hline EML-L & $47.017 \pm 1.122^{* *}$ & $50.529 \pm 1.814^{* *}$ & $130.134 \pm 11.987$ & $0.020 \pm 0.001 * *$ \\
\hline EML-M & $75.490 \pm 3.565^{* *}$ & $80.769 \pm 3.684^{* *}$ & $127.351 \pm 15.067$ & $0.012 \pm 0.001^{* *}$ \\
\hline EML-H & $86.140 \pm 2.613^{* *}$ & $91.058 \pm 3.291^{* *}$ & $121.108 \pm 13.126$ & $0.011 \pm 0.001 * *$ \\
\hline
\end{tabular}

* Significantly different with $P<0.05$ versus control group.

** Significantly different with $P<0.01$ versus control group.
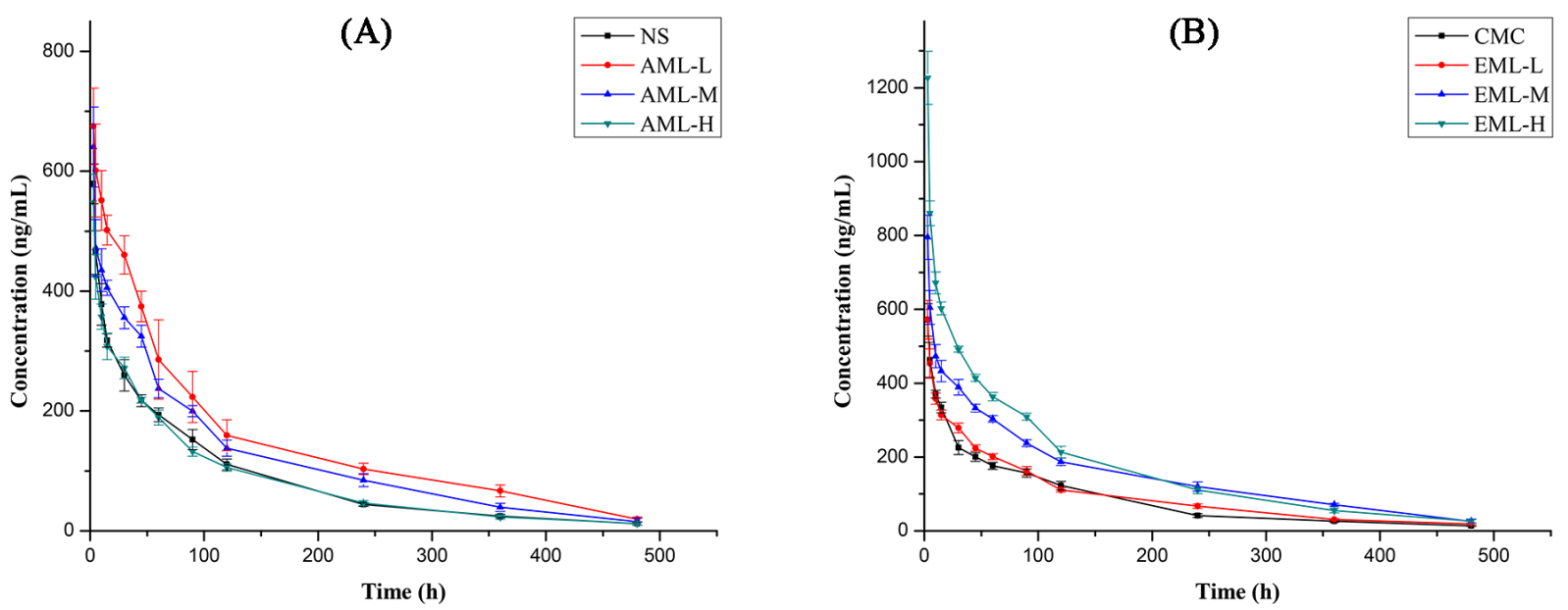

FIGURE 4 - Mean plasma concentration-time profiles of dextromethorphan in rats pre-treated with AML (A) and EML (B).

\subsubsection{Effect of mulberry leaf extracts on CYP2C19}

CYP2C19 activity was determined by comparing the pharmacokinetic profiles of omeprazole between control and mulberry leaf extract-treated groups. The effects of the low, medium and high doses of extract on the pharmacokinetics of omeprazole in rats are listed in Table VI. Mean plasma concentration-time curves of omeprazole are presented in Figure 5. AUC $(0-t)$ and $\mathrm{AUC}(0-\infty)$ values of omeprazole were significantly 
increased and CL significantly decreased in the EML-M and EML-H pretreatment groups, compared to CMC. On the other hand, the pharmacokinetic parameters of omeprazole were not significantly different in EML-L and all AML-treated rats relative, compared to the respective control groups.

TABLE VI - Main pharmacokinetic parameters of omeprazole in rats $(n=6$, mean \pm SD)

\begin{tabular}{lcccc}
\hline Group & $\begin{array}{c}\text { AUC(0-t) } \\
(\mathbf{m g} / \mathbf{L} \cdot \mathbf{m i n})\end{array}$ & $\begin{array}{c}\mathbf{A U C}(\mathbf{0}-\infty) \\
(\mathbf{m g} / \mathbf{L} \cdot \mathbf{m i n})\end{array}$ & $\begin{array}{c}\mathbf{t}_{\mathbf{1} / 2} \\
(\mathbf{m i n})\end{array}$ & $\begin{array}{c}\mathbf{C L} \\
(\mathbf{L} / \mathbf{m i n} / \mathbf{k g})\end{array}$ \\
\hline NS & $41.766 \pm 2.114$ & $42.153 \pm 2.049$ & $18.638 \pm 1.269$ & $0.024 \pm 0.001$ \\
AML-L & $40.340 \pm 1.779$ & $40.635 \pm 1.839$ & $17.279 \pm 1.978$ & $0.025 \pm 0.001$ \\
AML-M & $41.663 \pm 1.554$ & $41.923 \pm 1.566$ & $16.701 \pm 0.891$ & $0.024 \pm 0.001$ \\
AML-H & $41.855 \pm 0.899$ & $42.085 \pm 0.920$ & $16.339 \pm 1.049$ & $0.024 \pm 0.001$ \\
CMC & $42.190 \pm 2.352$ & $42.498 \pm 2.283$ & $18.437 \pm 1.414$ & $0.024 \pm 0.002$ \\
EML-L & $45.379 \pm 2.789$ & $45.783 \pm 2.801$ & $18.838 \pm 1.361$ & $0.022 \pm 0.001$ \\
EML-M & $65.903 \pm 4.009 * *$ & $66.328 \pm 4.089 * *$ & $18.442 \pm 1.130$ & $0.015 \pm 0.001 * *$ \\
EML-H & $72.115 \pm 4.078^{* *}$ & $72.820 \pm 4.240^{* *}$ & $21.783 \pm 3.517$ & $0.014 \pm 0.001 * *$ \\
\hline
\end{tabular}

* Significantly different with $P<0.05$ versus control group.

** Significantly different with $P<0.01$ versus control group.
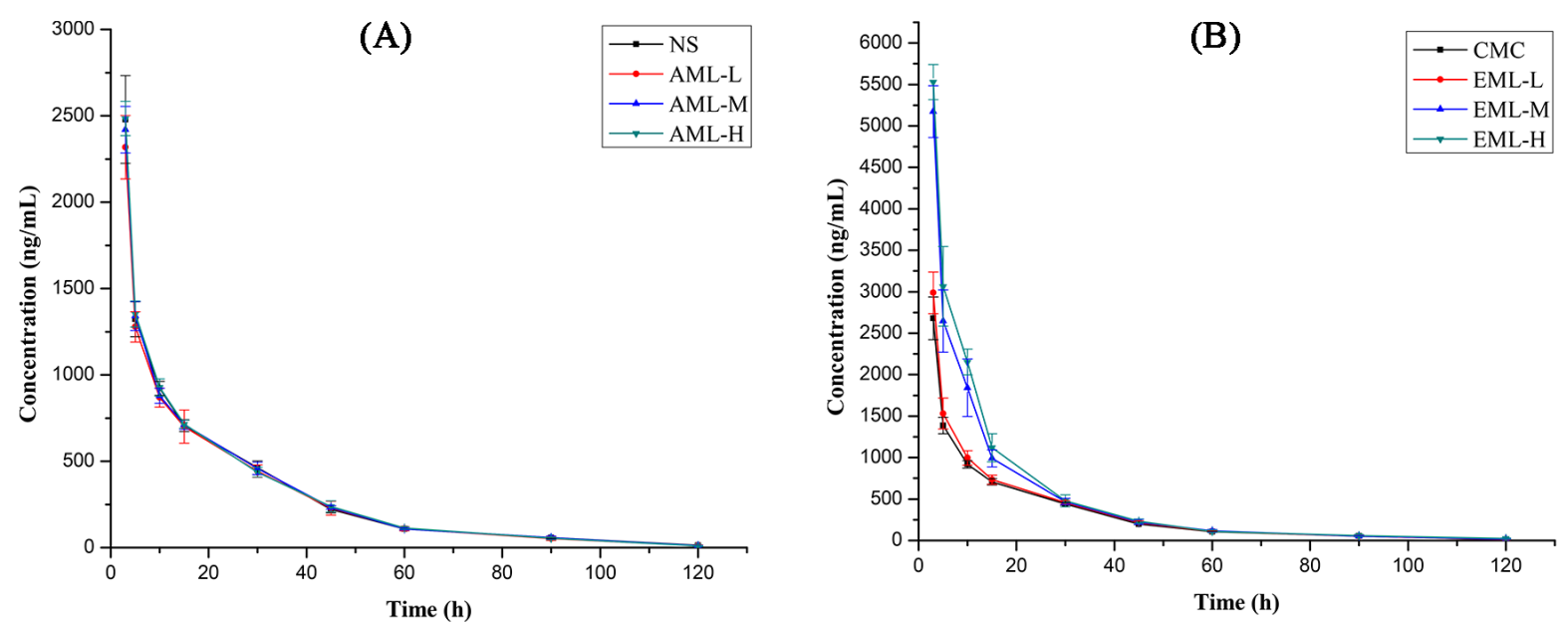

FIGURE 5 - Mean plasma concentration-time profiles of omeprazole in rats pre-treated with AML (A) and EML (B). 


\subsubsection{Effects of mulberry leaf extracts on CYP2C8}

The pharmacokinetic profiles of amodiaquine after mulberry leaf extract treatment were used to determine CYP2C8 activity. The mean plasma concentration-time curves of amodiaquine are presented in Figure 6 and the corresponding pharmacokinetic parameters listed in Table VII. The $\mathrm{AUC}_{(0-\mathrm{t})}$ and $\mathrm{AUC}_{(0-\infty)}$ values of amodiaquine were increased while CL was decreased to a significant extent, compared to NS, in the AML-M and AML-H pretreatment groups,. In contrast, no apparent influence of AML-L on these parameters was evident, indicating that middle and high doses of AML have the potential to inhibit rat CYP2C8 activity in vivo. No obvious effects on $\mathrm{AUC}_{(0-\mathrm{t})}, \mathrm{AUC}_{(0-\infty)}$ and $\mathrm{CL}$ values were observed at all EML doses relative to the control group.
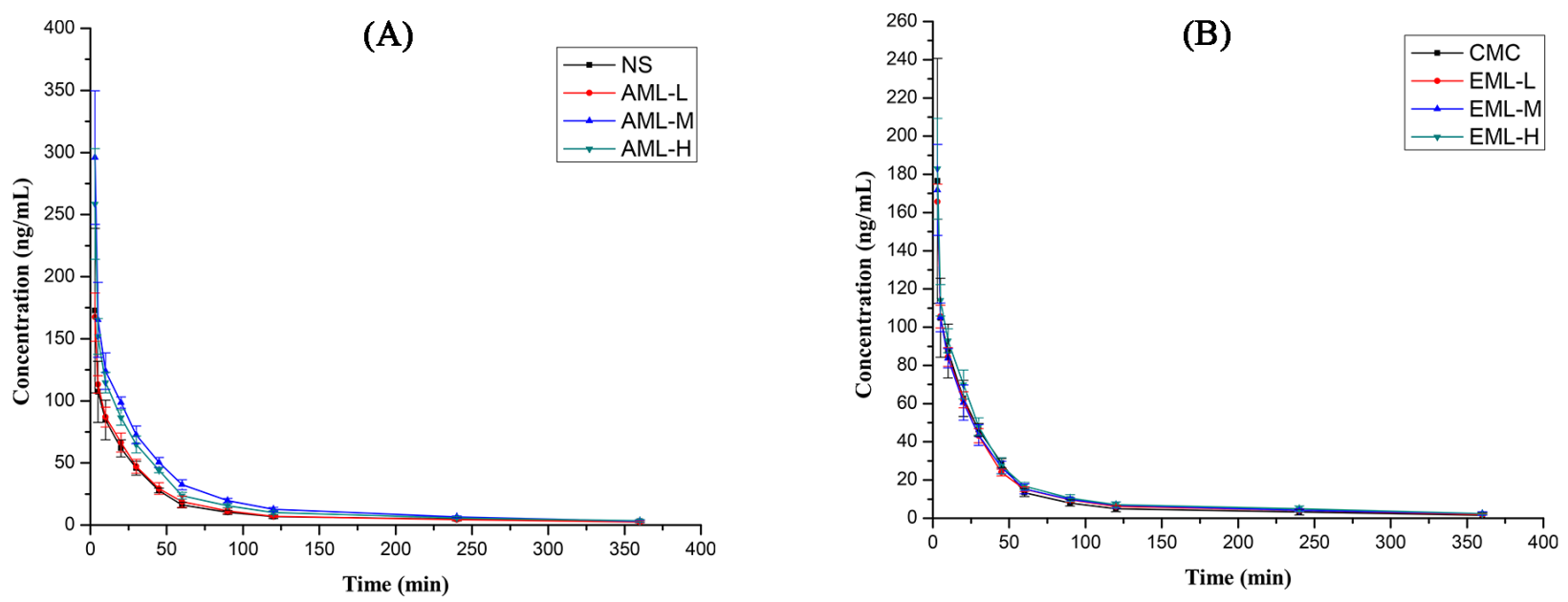

FIGURE 6 - Mean plasma concentration-time profiles of amodiaquine in rats pre-treated with AML (A) and EML (B).

TABLE VII - Main pharmacokinetic parameters of amodiaquine in rats $(n=6$, mean \pm SD)

\begin{tabular}{|c|c|c|c|c|}
\hline Group & $\begin{array}{c}\mathrm{AUC}(0-\mathrm{t}) \\
(\mathrm{mg} / \mathrm{L} \cdot \mathrm{min})\end{array}$ & $\begin{array}{c}\operatorname{AUC}(0-\infty) \\
(\mathrm{mg} / \mathrm{L} \cdot \min )\end{array}$ & $\begin{array}{c}\mathbf{t}_{1 / 2} \\
(\mathbf{m i n})\end{array}$ & $\begin{array}{c}\mathrm{CL} \\
(\mathrm{L} / \mathrm{min} / \mathrm{kg})\end{array}$ \\
\hline NS & $5.179 \pm 0.553$ & $5.814 \pm 0.869$ & $123.915 \pm 31.112$ & $0.176 \pm 0.028$ \\
\hline AML-L & $5.322 \pm 0.573$ & $5.828 \pm 0.754$ & $135.714 \pm 52.193$ & $0.174 \pm 0.021$ \\
\hline AML-M & $8.554 \pm 0.827^{* *}$ & $8.975 \pm 0.965^{* *}$ & $96.326 \pm 30.494$ & $0.112 \pm 0.012 *$ \\
\hline AML-H & $7.375 \pm 0.398^{* *}$ & $7.862 \pm 0.474 * *$ & $112.004 \pm 39.835$ & $0.128 \pm 0.008^{*}$ \\
\hline $\mathrm{CMC}$ & $4.696 \pm 0.436$ & $4.950 \pm 0.878$ & $113.642 \pm 17.287$ & $0.173 \pm 0.029$ \\
\hline EML-L & $4.818 \pm 0.343$ & $5.093 \pm 0.409$ & $103.372 \pm 11.592$ & $0.197 \pm 0.015$ \\
\hline EML-M & $4.964 \pm 0.676$ & $5.502 \pm 0.941$ & $145.184 \pm 35.558$ & $0.186 \pm 0.030$ \\
\hline EML-H & $5.423 \pm 0.665$ & $5.852 \pm 0.678$ & $126.025 \pm 26.808$ & $0.173 \pm 0.018$ \\
\hline
\end{tabular}

* Significantly different with $P<0.05$ versus control group.

** Significantly different with $P<0.01$ versus control group. 


\subsection{Effects of mulberry leaf extracts on mRNA expression of hepatic CYP enzymes}

\subsubsection{Effects of AML on mRNA expression of hepatic CYP enzymes}

The effects of all three doses of AML on CYP3A4, CYP1A2, CYP2D6, CYP2C19 and CYP2C8 mRNA expression are presented in Figure 7. After intragastric administration of AML for 14 days, the CYP3A4 mRNA level in the AML-L, AML-M, and AML-H groups was significantly increased by $0.23-, 0.33$ - and 1.01-fold, compared to that in NS, respectively. The CYP1A2 level in the AML-L, AML-M, and AML-H groups was significantly decreased by $0.25-, 0.40$ - and 0.38 -fold, compared to that in NS, respectively. The CYP2D6 level in the AML-M and AML-H groups was markedly decreased by 0.14 - and 0.22 -fold relative to that in NS, respectively. No significant differences in CYP2D6 mRNA expression were evident between the AML-L and NS groups. CYP2C19 mRNA expression in the three treatment groups was comparable to that in NS. The CYP2C8 mRNA level in the AML-M and AML-H groups was markedly decreased by $0.36-$ fold and 0.55 -fold that in NS, respectively, while no significant differences were observed between AML-L and NS groups.

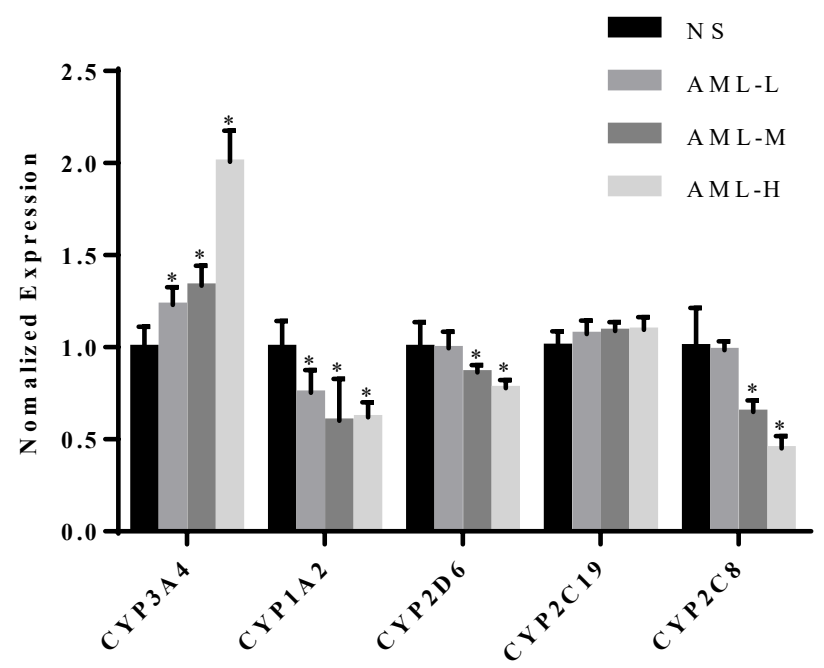

FIGURE 7 - Effect of AML on mRNA expression of CYP3A1, CYP1A2, CYP2D2, CYP2C11 and CYP2C7 in rats $(n=6)$. $* \mathrm{P}<0.05$ versus control group.

\subsubsection{Effects of EML on mRNA expression of hepatic CYP enzymes}

The effects of EML at all three doses on CYP3A4, CYP1A2, CYP2D6, CYP2C19 and CYP2C8 mRNA levels are presented in Figure 8. Compared with the CMC group, CYP3A4 mRNA expression in rat liver was significantly increased by 0.32 -fold, 0.88 -fold, and 0.93-fold in the EML-L, EML-M, and EML-H groups, respectively. CYP1A2 mRNA in the EML-M and EML-H groups was decreased by 0.28 -fold and 0.29 -fold while in EML-L, expression was not significantly different relative to that in CMC. CYP2D6 levels in EML-L, EML-M and EML-H groups were markedly decreased by 0.11-, 0.23and 0.25-fold, compared to CMC. Similarly, EML inhibited CYP2C19 mRNA expression, with a 0.38 -fold and 0.53fold increase observed in the EML-M and EML-H groups, respectively. No significant differences in CYP2C19 mRNA expression were evident between the EML-L and CMC groups. The mRNA levels of CYP2C 8 in all the treatment groups were comparable to that in $\mathrm{CMC}$.

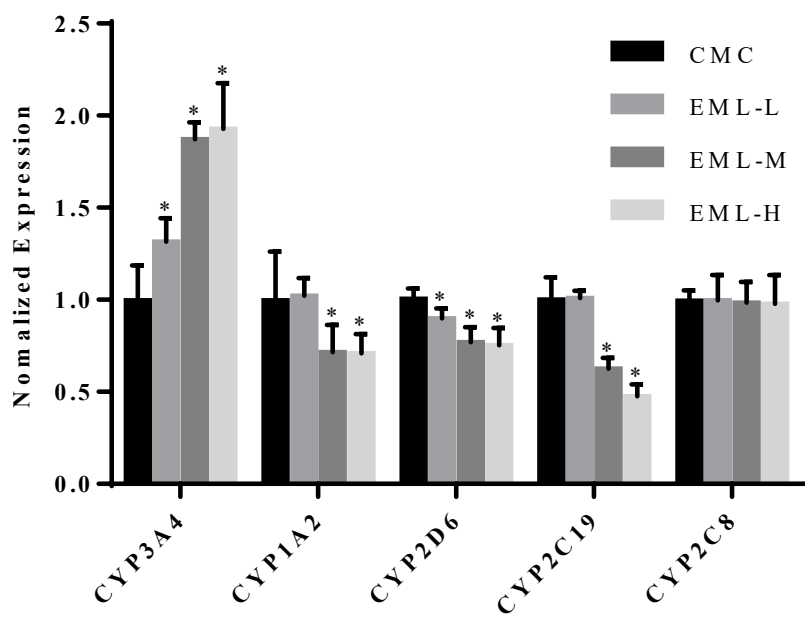

FIGURE 8 - Effect of EML on mRNA expression of CYP3A1, CYP1A2, CYP2D2, CYP2C11 and CYP2C7 in rats $(\mathrm{n}=6)$. $* \mathrm{P}<0.05$ versus control group.

\section{DISCUSSION}

Mulberry leaves, one of the most commonly used agents in herbaceous traditional Chinese medicines, are usually considered for the treatment of diabetes mellitus and its complications. However, determination of the 
potential effects of herbs on CYP activities is necessary for their safe and effective application. Here, we systematically investigated the effects of mulberry leaf extracts on the activities and mRNA expression levels of five CYP450 isoforms in a rat model.

The effects of mulberry leaf extracts on mRNA levels of rat CYP3A4, CYP1A2, CYP2D6, CYP2C19 and CYP2C8 and pharmacokinetics of five corresponding probe drugs in vivo were characterized. The clinical dose of mulberry leaf used was 5-10 g. On the basis of dose conversion between human and rat, we selected $1 \mathrm{~g} / \mathrm{kg}$ mulberry leaf as the low dose, $2 \mathrm{~g} / \mathrm{kg}$ as the medium dose, and $4 \mathrm{~g} / \mathrm{kg}$ as the high dose for administration over 14 consecutive days and evaluated the effects on five CYP enzymes in rats.

CYP3A4 is the most important enzyme in liver involved in the metabolism of various drugs for treatment of diabetes, such as gliquidone, glyburide, and atorvastatin (He et al., 2014; Zhou et al., 2010; Berta et al., 2014). Our data on the potential influence of mulberry leaf extracts (AML and EML) on activities of rat CYP3A4 suggest that treatment with the extracts reduces the blood concentrations of drugs metabolized by CYP3A4, thus highlighting the need for attempts to reduce the risk of failure in potential clinical applications when drugs are administered together with substrates of CYP3A4.

CYP1A 2 accounts for $13 \%$ of the total CYP content and is involved in the metabolism of $8-10 \%$ drugs on the market (Guengerich, FP, Turvy, CG, 1991; Jiang et al., 2017; Kapoor et al., 2006). Although low levels of CYP2D6 are expressed in liver accounting for $\sim 4 \%$ total CYP, this enzyme is involved in the biotransformation of $30 \%$ currently available drugs. In our experiments, both CYP1A2 and CYP2D6 activities were significantly inhibited by mulberry leaf (AML and EML) extracts after multiple oral administration in rats, suggesting that combination treatment with mulberry leaves and substrate drugs metabolized by human CYP1A2 and CYP2D6 could result in significant herb-drug interactions.

The CYP2C enzymes comprise the most complex subfamily accounting for $20 \%$ of the total hepatic CYP content and interact with $\sim 16 \%$ drugs available in the market at present (Wang et al., 2018). CYP2C 8 is the major form, which represents $35 \%$ of the total CYP2C family, and $2 \mathrm{C} 19(1 \%)$ is the minor CYP2C isoform (Romkes et al., 1991). CYP2C8 plays a critical role in the metabolism of several antidiabetic agents, such as original, repaglinide, rosiglitazone and pioglitazone (Jaakkola et al., 2006; Gan et al., 2010; Tornio et al., 2012). According to our results, middle- and high-dose AML suppressed CYP2C8 activity to a significant extent. Thus, prior to co-administration of AML with drugs metabolized by CYP2C8 in the clinic, it is important to focus on the potential sideeffects of AML-drug interactions. Interestingly, EML had no significant effects on CYP2C8 activity, even after long-term administrations in rats. Additionally, previous studies have reported CYP2C19 as the major CYP enzyme involved in the metabolism of gliclazide (Elliot et al., 2007). Our experiments disclosed significant inhibition of CYP2C19 activity by EML after 14-day administration in rats. Therefore, upon co-administration with EML, drugs metabolized by human CYP2C19 may require dose adjustment to avoid undesirable herb-drug interactions (CYP2C19 activity was not significantly affected by AML).

A recent reported highlighted that the majority $(72.8 \%)$ of diabetic patients use herbal medicines as alternative therapies (Gupta et al., 2017). Herbal extracts are perceived as safe alternatives to achieve the ultimate goal of reducing toxicity and enhancing efficacy (Cao L, Kwara A, Greenblatt DJ, 2017). However, it is important to note that most herbal extracts have complex bioactive phytoconstituents (Rodeiro et al., 2009; Pan et al., 2011) and concurrent treatment of diabetes with multiple drugs often results in complications. Herbal extracts thus have the potential to cause HDI. In recent years, numerous studies have reported interactions between herbs and drugs. Here, we examined the effects of mulberry leaf extracts on CYP enzyme activities in a rat model in vivo. Our results disclosed promotory effects of mulberry leaf extracts (AML and EML) on CYP3A4 activity and differential inhibitory effects on other CYP enzymes (CYP2C8, CYP2C19, CYP2D6, and CYP1A2) in rat, highlighting the importance of further research focus on the potential occurrence of HDIs when mulberry leaves and CYP enzyme-metabolizing drugs are used in combination.

In conclusion, our findings emphasize the need for caution in co-administering mulberry leaves and drugs metabolized by CYP450 enzymes, including CYP3A4, CYP2C8, CYP2C19, CYP2D6, and CYP1A2, to prevent potential adverse reactions. Our results provide a basis for the rational selection of drugs for combination with mulberry leaf therapy in clinical practice and formulation of guidelines for their safe and effective use.

\section{ACKNOWLEDGMENTS}

This work was supported by the National Natural Science Foundation of China (No. 81773988) and Anhui 
Province Programs for Science and Technology Research and Development (No. 1301042099).

\section{CONFLICTS OF INTEREST}

The authors have no conflicts of interest to declare.

\section{ETHICAL APPROVAL}

The study was approved by the Animal Ethics Committee of Anhui University of Chinese Medicine (Anhui, China). All procedures involving animals adhered to the ethical standards of the institution in which the studies were conducted.

\section{REFERENCES}

Berta E, Harangi M, Zsíros N, Nagy EV, Paragh G, Bodor M. Effect of thyroid hormone status and concomitant medication on statin induced adverse effects in hyperlipidemic patients. Pharmazie. 2014;69(6):420-423.

Cai R, Liu M, Hu Y, Melton BL, Matheny ME, Xu H, et al. Identification of adverse drug-drug interactions through causal association rule discovery from spontaneous adverse event reports. Artif Intell Med. 2017;76:7-15.

Cao L, Kwara A, Greenblatt DJ. Metabolic interactions between acetaminophen (paracetamol) and two flavonoids, luteolin and quercetin, through in-vitro inhibition studies. J Pharm Pharmacol. 2017;69(12):1762-1772.

Elliot DJ, Suharjono, Lewis BC, Gillam EM, Birkett DJ, Gross AS, et al. Identification of the human cytochromes P450 catalysing the rate-limiting pathways of gliclazide elimination. Br J Clin Pharmacol. 2007;64(4):450-457.

Gan J, Chen W, Shen H, Gao L, Hong Y, Tian Y, et al. Repaglinide-gemfibrozil drug interaction:inhibition of repaglinide glucuronidation as a potential additional contributing mechanism. Br J Clin Pharmacol. 2010;70(6):870880 .

Guengerich FP, Turvy CG. Comparison of levels of several human microsomal cytochrome P450 enzymes and epoxide hydrolase in normal and disease states using immunochemical analysis of surgical liver samples. J Pharmacol Exp Ther. 1991;256(3):1189-1194.

Guo YJ, Zheng SL. Effect of myricetin on cytochrome P450 isoforms CYP1A2, CYP2C9 and CYP3A4 in rats. Pharmazie. 2014;69(4):306-310.
Gupta RC, Chang D, Nammi S, Bensoussan A, Bilinski $\mathrm{K}$, Roufogalis BD. Interactions between antidiabetic drugs and herbs: an overview of mechanisms of action and clinical implications. Diabetol Metab Syndr. 2017;9:59.

MSA, Chilkunda ND. Morus alba leaf bioactives modulate Peroxisome proliferator activated receptor $\gamma$ in the kidney of diabetic rat and impart beneficial effect. J Agric Food Chem. 2018;66(30):7923-7934.

He F, Li Y, Zeng C, Xia C, Xiong Y, Zhang H,et al. Contribution of cytochrome $\mathrm{P} 450$ isoforms to gliquidone metabolism in rats and human. Xenobiotica. 2014;44(3):229-234.

Jaakkola T, Laitila J, Neuvonen PJ, Backman JT. Pioglitazone is metabolised by CYP2C8 and CYP3A4 in vitro: potential for interactions with CYP2C8 inhibitors. Basic Clin Pharmacol Toxicol. 2006;9(1):44-51.

Jiang B, Meng L, Zhang F, Jin X, Zhang G. Enzyme-inducing effects of berberine on cytochrome P450 1A2 in vitro and in vivo. Life Sciences. 2017;189:1-7.

Joh B, Jeon ES, Lim SH, Park YL, Park W, Chae H. Intercultural usage of mori folium: comparison review from a Korean medical perspective. Evid Based Complement Alternat Med. 2015;2015:379268.

Kapoor N, Pant AB, Dhawan A, Dwievedi UN, Seth PK, Parmar D. Cytochrome P450 1A isoenzymes in brain cells: expression and inducibility in cultured rat brain neuronal and glial cells. Life Sciences. 2006;79(25):2387-2394.

Liu R, AbdulHameed MDM, Kumar K, Yu X, Wallqvist A, Reifman J. Data-driven prediction of adverse drug reactions induced by drug-drug interactions. BMC Pharmacol Toxicol. 2017; 18(1):44.

Pan Y, Abd-Rashid BA, Ismail Z, Ismail R, Mak JW, Pook $\mathrm{PC}$, et al. In vitro determination of the effect of andrographis paniculata extracts and andrographolide on human hepatic cytochrome P450 activities. J Nat Med. 2001; 65(3-4):440-7.

Reginald EF. Probing the world of cytochrome P450 enzymes. Molecular Interventions. 2004;4(3):157-62.

Rendic S, Di Carlo FJ. Human cytochrome P450 enzymes: a status report summarizing their reactions, substrates, inducers, and inhibitors. Drug Metab Rev. 1997; 29(1-2):413580 .

Rodeiro I, Donato MT, Jimenez N, Garrido G, Molina-Torres J, Menendez R, et al. Inhibition of human P450 enzymes by natural extracts used in traditional medicine. Phytother Res. 2009; 23(2): 279-82. 
Effects of mulberry leaf extracts on activity and mRNA expression of five cytochrome P450 enzymes in rat

Romkes M, Faletto MB, Blaisdell JA, Raucy JL, Goldstein JA. Cloning and expression of complementary DNAs for multiple members of the human cytochrome P450IIC subfamily. Biochemistry. 1991;30(13):3247-3255.

Tornio A, Niemi M, Neuvonen PJ, Backman JT. Drug interactions with oral antidiabetic agents: pharmacokinetic mechanisms and clinical implications. Trends Pharmacol Sci. 2012;33(6):312-322.

Wang GQ, Zhu L, Ma ML, Chen XC, Gao Y, Yu TY, et al. Mulberry 1-Deoxynojirimycin inhibits adipogenesis by repression of the ERK/PPAR $\gamma$ signaling pathway in porcine intramuscular adipocytes. Journal of Agricultural and Food Chemistry. 2015;63(27):6212-6220.

Wang J, Zhai T, Chen Y. Effects of honokiol on CYP450 activity and transporter mRNA expression in type 2 diabetic rats. Int J Mol Sci. 2018;19(3):815-825.

Lu YY, Du ZY, Li Y, Wang JL, Zhao MB, Jiang Y, et al. Effects of Baoyuan decoction, a traditional Chinese medicine formula, on the activities and mRNA expression of seven CYP isozymes in rats. J Ethnopharmacol. 2018,255:327-335.

Zhang Y, Ren CJ, Lu GB, Mu ZM, Cui WZ, Gao HJ, et al. Antidiabetic effect of mulberry leaf polysaccharide by inhibiting pancreatic islet cell apoptosis and ameliorating insulin secretory capacity in diabetic rats. Int Immunopharmacol. 2014;22(1):248-57.

Zheng XK, Cao YG, Ke YY, Zhang YL, Li F, Gong JH, et al. Phenolic constituents from the root bark of Morus alba L. and their cardioprotective activity in vitro. Phytochemistry. 2017;135:128-34.

Zhou L, Naraharisetti SB, Liu L, Wang H, Lin YS, Isoherranen $\mathrm{N}$, et al. Contributions of human cytochrome P450 enzymes to glyburide metabolism. Biopharm Drug Dispos. 2010;31(4):228-42.

Zhou SF, Liu JP, Chowbay B. Polymorphism of human cytochrome P450 enzymes and its clinical impact. Drug Metab Rev. 2009;41(2):89-295.

Received for publication on $11^{\text {st }}$ December 2018 Accepted for publication on $01^{\text {st }}$ April 2019 